\title{
Influence of portal vein occlusion on portal flow and liver elasticity in an animal model
}

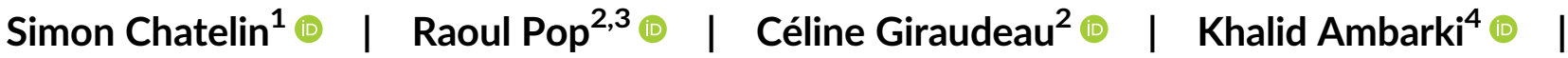

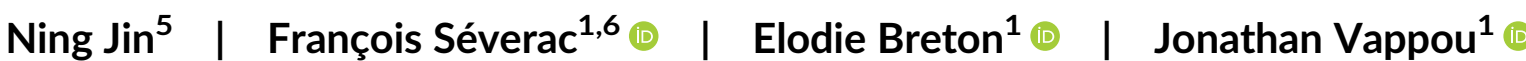

${ }^{1}$ ICube, CNRS UMR 7357, University of Strasbourg, Strasbourg, France

${ }^{2} \mathrm{HHU}$-Strasbourg, Institute for Image-Guided Surgery, Strasbourg, France

${ }^{3}$ Interventional Neuroradiology Department, University Hospital of Strasbourg, Strasbourg, France

${ }^{4}$ Siemens Healthcare SAS, Saint Denis, France

${ }^{5}$ Siemens Medical Solutions USA, Inc., Chicago, Illinois, USA

${ }^{6}$ Public Healthcare Department, University Hospitals Strasbourg, Strasbourg, France

\section{Correspondence}

Simon Chatelin, ICube, CNRS UMR 7357, University of Strasbourg, Bat. IHU, 1 Place de I'hôpital, 67091, Strasbourg Cedex, France.

Email: schatelin@unistra.fr

Funding information

Agence Nationale de la Recherche, Grant/

Award Number: ANR-10-IAHU-02
Hepatic fibrosis causes an increase in liver stiffness, a parameter measured by elastography and widely used as a diagnosis method. The concomitant presence of portal vein thrombosis (PVT) implies a change in hepatic portal inflow that could also affect liver elasticity. The main objective of this study is to determine the extent to which the presence of portal occlusion can affect the mechanical properties of the liver and potentially lead to misdiagnosis of fibrosis and hepatic cirrhosis by elastography. Portal vein occlusion was generated by insertion and inflation of a balloon catheter in the portal vein of four swines. The portal flow parameters peak flow (PF) and peak velocity magnitude (PVM) and liver mechanical properties (shear modulus) were then investigated using 4D-flow MRI and MR elastography, respectively, for progressive obstructions of the portal vein. Experimental results indicate that the reduction of the intrahepatic venous blood flow (PF/PVM decreases of $29.3 \% / 8.5 \%, 51.0 \% / 32.3 \%$ and $83.3 \% / 53.6 \%$, respectively) measured with $50 \%$, $80 \%$ and $100 \%$ obstruction of the portal vein section results in a decrease of liver stiffness by $0.8 \% \pm 0.1 \%, 7.7 \% \pm 0.4 \%$ and $12.3 \% \pm 0.9 \%$, respectively. While this vascular mechanism does not have sufficient influence on the elasticity of the liver to modify the diagnosis of severe fibrosis or cirrhosis (F4 METAVIR grade), it may be sufficient to attenuate the increase in stiffness due to moderate fibrosis (F2-F3 METAVIR grades) and consequently lead to false-negative diagnoses with elastography in the presence of PVT.

\section{KEYWORDS}

animal model study, flow quantification, hepatobiliary, MR elastography

\section{1 | INTRODUCTION}

Portal vein thrombosis (PVT) is a partial or complete occlusion by an intraluminal thrombus of the blood flow within the portal vein. The lifetime risk of developing PVT has been estimated to be around $1 \% .{ }^{1}$ The main consequence of PVT is portal hypertension and its associated complications. ${ }^{2}$ PVT has local origin in $70 \%$ of cases (with abdominal cancers, focal inflammatory lesions, injury to the portal venous system and cirrhosis being the most frequent risk factors) and systemic origins in $30 \%$ of cases (congenital or acquired deficiencies), and it is a common complication in patients with liver disease. ${ }^{3}$ 
A high prevalence of PVT is observed in the cirrhotic population, ${ }^{4}$ increasing with the severity of cirrhosis from $1 \%$ in well-compensated cirrhosis to $7.5 \%-16 \%$ in advanced cirrhosis ${ }^{5-8}$ and $35 \%$ in cirrhotic patients with hepatocellular carcinoma. ${ }^{9}$ Reduction in the portal venous flow velocity is to date one of the most important predictive variables for PVT outcomes in patients with cirrhosis. ${ }^{10} \mathrm{~A}$ link between coagulation activity and progressive liver fibrosis has also been shown, most likely due to a reaction to tissue ischemic damage. ${ }^{11}$

Liver elasticity measurement using elastography is today one of the most pertinent predictive factors as an alternative to liver biopsy, allowing estimation of the fibrosis stage and presence of cirrhosis. ${ }^{12-17}$ The liver stiffens globally as the fibrotic and cirrhotic stage increases. This stiffening is mainly due to modifications of the hepatic tissue, namely by the development of regenerative nodules surrounded by fibrous bands. Despite good agreement between elasticity measurement and fibrosis stage, a recent case study has reported PVT as a potential source of false-negative diagnoses of fibrosis using elastography. ${ }^{18}$ The authors suggest that a decrease of the stiffness due to PVT could have compensated the stiffening usually observed in fibrotic livers. Recent reports of a decreased liver stiffness caused by reduced intrahepatic venous blood flow would reinforce this assumption. ${ }^{19,20}$ Thus, the modification induced in the liver mechanical properties by fibrosis, mainly reflecting the alteration in the constitutive hepatic tissue, may be attenuated by portal flow modifications in the presence of PVT.

In the present study, we proposed an original preclinical investigation of the consequences of intraluminal portal vein occlusion on both portal flow and liver elasticity, in order to answer the following question: To what extent is the presence of PVT likely to influence liver elasticity, and thereby potentially degrade the diagnosis of liver fibrosis or cirrhosis by elastography? In this study, modifications of the venous portal inflow were obtained in vivo in four animal subjects (swine) through various levels of portal vein obstruction with a balloon catheter. For each level of portal vein obstruction, portal vein flow and liver elasticity were quantified noninvasively using 4D-flow MRI and MR-elastography (MRE), respectively. The in vivo, noninvasive quantification of the vascular and mechanical effects of portal obstruction on the liver were thus obtained.

\section{2 | MATERIALS AND METHODS}

The study was conducted on four female swines, weighing 27, 25, 27 and $30 \mathrm{~kg}$ for subjects \# 1 to \#4, respectively. Anesthesia was induced using intravenous Zoletil ( $20 \mathrm{cc}$, Virbac) and was maintained using $2 \%$ isoflurane (Forene, AbbVie). Approval from the institutional animal care committee was obtained. All the experiments were performed in agreement with the European Community Council Directive (010/63/UE) and in accordance with national animal experiment regulations (APAFIS \#14092-2018031513247711 v1). A surgical procedure was first performed under angio-scanner monitoring to introduce the balloon catheter for portal venous flow obstruction, followed by the MRI protocol.

\subsection{Control of the portal flow}

Occlusion of the portal vein was obtained using a balloon catheter (XXL Vascular, Boston Scientific), with dimensions adapted to the portal vein diameter, measured with $\mathrm{x}$-ray fluoroscopy. Percutaneous access to an intrahepatic branch of the portal vein was performed under ultrasound (Acuson S3000, Siemens Healthcare) and x-ray fluoroscopy guidance (Artis Zeego system, Siemens Healthcare). The balloon catheter was then introduced in the main trunk of the portal vein under fluoroscopy guidance. The inflation volume in the balloon was calibrated under fluoroscopy for each predetermined degree of portal occlusion ( $0 \%, 50 \%, 80 \%$ and $100 \%$ of the portal vein diameter). For that purpose, a $1: 1$ iodine contrast (Visipaque 270, GE Healthcare) in normal saline solution was used for balloon inflation. Representative angiography images for all four animals are provided in Figure 1B, for a balloon catheter dilatation of $80 \%$ of the intraluminal portal vein section.

\section{2 | MRI procedure}

MRI acquisitions were conducted in a 1.5-T MRI (MAGNETOM Aera, Siemens Healthcare) using the in-bore body coil, with the animal lying in supine position. Normal saline was used for balloon inflation. The inflation volume in the balloon catheter was varied to obtain successive intraluminal obstructions of approximately $0 \%$ (reference, no inflation), 80\%, 50\%, 0\% (control) and 100\% of the portal vein section. Actual obstruction levels were evaluated after the procedure in post-treatment of the SPIRAL-VIBE 3D MRI acquisitions. This experimental order makes it possible not only to avoid total occlusion, potentially risky for the animal, from the beginning of the procedure, but also to have a control point in the middle of the procedure, given that anesthesia or previous occlusions may have induced physiological effects leading to a variation in liver elasticity. For each inflation level of the balloon catheter, the following MRI sequences were successively acquired (according to the experimental protocol illustrated in Figure 1A):

1. LOCA: fast scout localizer images.

2. T1-VIBE: for precise positioning of the field of view (FOV) of the following sequences. 


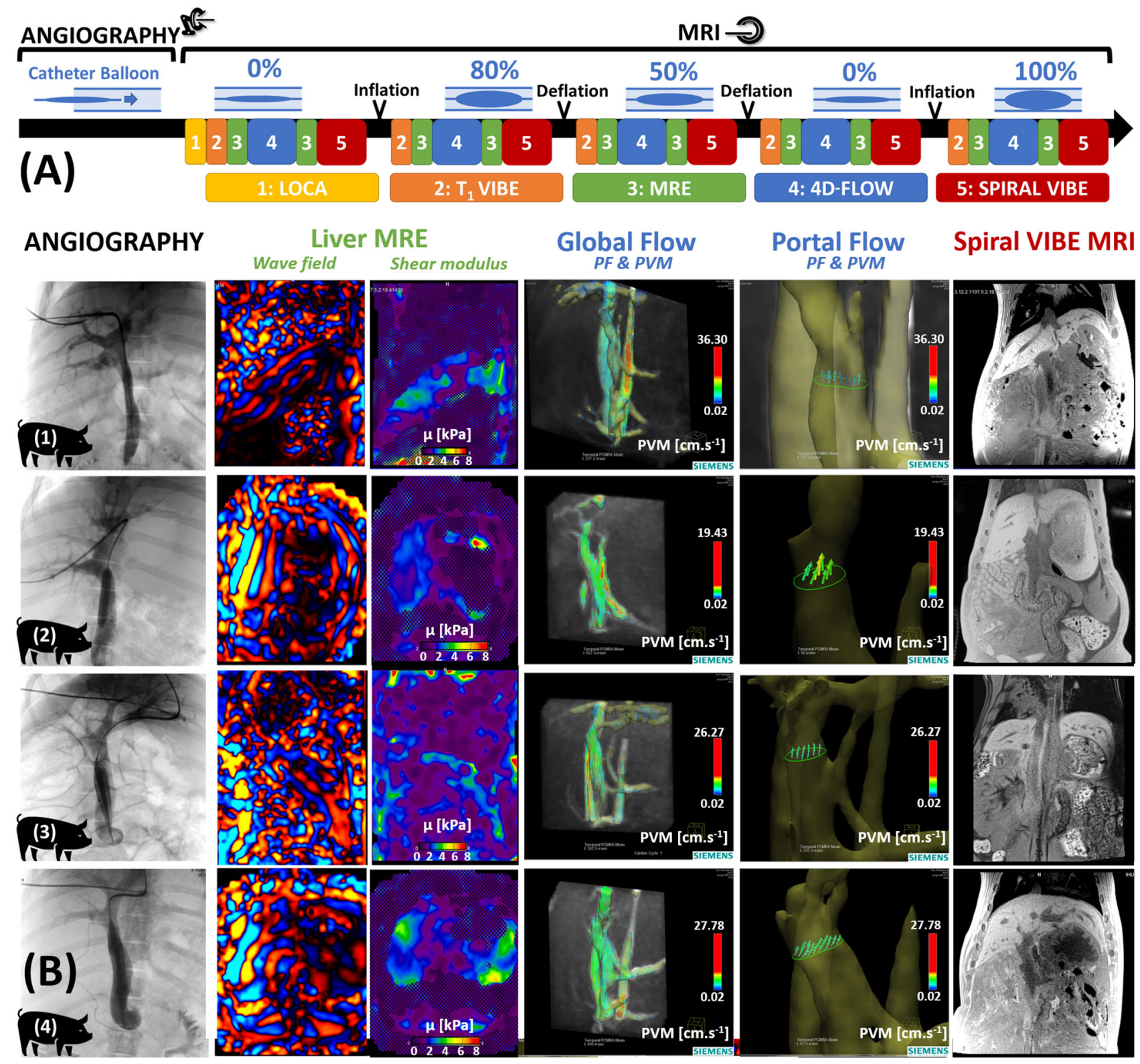

FIGURE 1 (A) Timeline of the experimental protocol. After positioning of the catheter through the portal vein under x-ray guidance, MRI acquisitions alternating 4D-flow, magnetic resonance elastography (MRE) and anatomical acquisitions were performed for successive dilation states of the balloon. (B) Representative angiography, 4D-flow, MRE wave, MRE stiffness map and SPIRAL-VIBE MRI images are presented for all four animal models. PF, peak flow; PVM, peak velocity magnitude

3. MRE \#1: these acquisitions aim at mapping and quantifying the liver elasticity. The MRE acquisitions were performed in the liver using a gradient recalled echo (GRE) sequence with flow-compensated motion sensitizing gradients and mechanical vibration at $60 \mathrm{~Hz}$ using a pneumatic exciter (Resoundant).

4. $4 D$-flow: the $4 D$-flow sequence aims at investigating over time in $3 D$ the peak flow (PF) and the peak velocity magnitude (PVM) in the portal vein and vena cava. Acquisitions were obtained using the research prototype 4D flow pulse sequence ${ }^{21}$ WIP785A (Siemens Healthcare). Flow parameters were quantified using the prototype "4DFlow v2.4" WIP postprocessing software (Siemens Healthcare). Phase antialiasing and motion tracking filtering were applied as post-treatment before flow quantifications. PF and PVM are estimated downstream of the balloon in the main trunk of the portal vein, halfway between the end of the balloon and the division into right and left portal branches.

5. MRE \#2: repeat.

6. SPIRAL-VIBE: the high-resolution images aim at measuring the diameter of both the balloon catheter and the portal vein. Acquisitions were performed using the UTE spiral 3D-GRE sequence with volumetric interpolation (VIBE). The intraluminal sectional vein obstruction was then calculated for each of the theoretical inflation states as the ratio of the balloon diameter to the portal vein diameter, measured in the SPIRAL-VIBE images. 
Images were acquired in the coronal plane (except for MRE images acquired in an oblique plane aligned against the inclination of the pneumatic vibrator, i.e. MRE images acquired perpendicular to the active face of the pneumatic vibrator). The main acquisition parameters are summarized in Table 1. The animal was under breath-hold (mechanical ventilation was stopped) during T1-VIBE and MRE acquisitions. The 4D-flow sequence was respiratory-gated using a pressure belt sensor. Because of the long acquisition time of the 4D-flow, the MRE acquisition was performed before and after the 4D-flow acquisition. The MRE shear modulus used in the analysis corresponds to the average of these two measurements. Representative angiography, 4D-flow, MRE waves, MRE stiffness maps and SPIRAL-VIBE MRI images are shown for all four animals in Figure 1B.

\section{3 | Analysis}

The liver stiffness and flow values were expressed as mean \pm standard deviation.

The statistical repeatability of MRE measurements was evaluated using R Core Team 2019 v.3.6. ${ }^{22}$ The within-subject coefficient of variation (wCV) was evaluated with 95\% lower limit and upper limit confidence intervals, including variance stabilizing transformation, ${ }^{23,24}$ and interpreted based on the criteria defined in. ${ }^{25}$

The individual correlation between the liver stiffness and the portal PVM or PF was calculated for each animal using Spearman's $r_{s}$ rank correlation coefficient to investigate potential monotonic relationships (statistical analysis was performed using the XLSTAT add-in; Addinsoft, Paris, France ${ }^{26}$ ).

\section{3 | RESULTS}

\subsection{Occlusion of the portal vein and flow quantification}

In addition to nominal balloon dimensions (diameter and length), the intraluminal sectional vein obstruction, balloon diameter and portal vein diameter measured by MRI are reported in Table 2. The measured sectional obstructions were close to the expected values (with a maximal difference of 3.3\%). Dextrocardia was observed in animal subject \#3 (Figure 1B).

Baseline PVM and PF were measured at initial $0 \%$ occlusion as $15.53 \pm 2.45 \mathrm{~cm} . \mathrm{s}^{-1}$ and $13.46 \pm 1.63 \mathrm{~mL} . \mathrm{s}^{-1}$, respectively, averaged over subjects. The individual evolutions of both the portal PF and PVM obtained from 4D-flow data are shown in Figure 2 (orange and blue dots, respectively) against the sectional obstruction level of the portal vein. Note that for readability, the scale for subject \#3 is different from the one for other subjects. It should be noted that for $100 \%$ occlusion, defined as the balloon diameter being equal to the intraluminal portal vein section, portal vein compliance (elasticity) ensures remnant portal flow. For nominal intraluminal occlusions of the portal vein of $50 \%$, $80 \%$ and $100 \%$, the mean decrease of the portal PF was $29.3 \%, 51.0 \%$ and $83.3 \%$, respectively, compared with the initial $0 \%$ occlusion reference. Similarly, the portal PVM was reduced by $8.5 \%, 32.3 \%$ and $53.6 \%$ for nominal intraluminal occlusions of $50 \%, 80 \%$ and $100 \%$ of the portal vein, respectively, compared with the initial $0 \%$ occlusion reference PVM. Compared with the second "control" $0 \%$ occlusion state, the mean decrease in portal PF and

TAB LE 1 Relevant acquisition parameters for the MRI sequences

\begin{tabular}{|c|c|c|c|c|}
\hline Parameter & T1-VIBE & MRE $60 \mathrm{~Hz}$ & 4D-flow & SPIRAL-VIBE \\
\hline TR/TE [ms] & $4.72 / 2.18$ & $50 / 23.75$ & $51.68 / 3.77$ & $4.57 / 0.05$ \\
\hline TT [ms] & - & - & 20 to 440 by 52.5 steps ( 9 frames) & - \\
\hline $\mathrm{FOV}[\mathrm{mm} \times \mathrm{mm}]$ & $243 \times 300$ & $239 \times 300$ & $284 \times 414$ & $300 \times 300$ \\
\hline Matrix & $156 \times 256$ & $204 \times 256$ reco pas acq & $106 \times 192$ & $288 \times 288$ \\
\hline Slice thickness [mm] & 2 & 5 & 2.5 & 1 \\
\hline Bandwidth frequency [Hz/pixel] & 350 & 260 & 450 & - \\
\hline Number of time frames & - & - & 11 & \\
\hline Encoding velocity $[\mathrm{cm} / \mathrm{s}]$ & - & - & 50 (in all directions) & - \\
\hline Duration [s] & 18 & 67 & $\sim 518$ & $\sim 278$ \\
\hline
\end{tabular}


TABLE 2 Individual intraluminal sectional vein occlusions as determined on the SPIRAL-VIBE images

\begin{tabular}{|c|c|c|c|c|c|c|}
\hline \multirow[b]{2}{*}{ Animal \# } & \multirow[b]{2}{*}{ Parameters } & \multicolumn{4}{|c|}{ Nominal intraluminal sectional vein occlusion [\%] } & \multirow[b]{2}{*}{ Nominal balloon diameter/length [mm] } \\
\hline & & $0 \%$ & $50 \%$ & $80 \%$ & $100 \%$ & \\
\hline \multirow[t]{3}{*}{1} & Balloon diameter $[\mathrm{mm}]$ & 0.6 & 5.8 & 7.9 & 9.1 & \multirow[t]{3}{*}{$9 / 40$} \\
\hline & Portal vein diameter $[\mathrm{mm}]$ & 8.7 & 7.9 & 8.9 & 9.1 & \\
\hline & Sectional obstruction [\%] & 0.4 & 52.5 & 79.8 & 100.0 & \\
\hline \multirow[t]{3}{*}{2} & Balloon diameter $[\mathrm{mm}]$ & 1.0 & 8.3 & 10.2 & 12.3 & \multirow[t]{3}{*}{$12 / 40$} \\
\hline & Portal vein diameter $[\mathrm{mm}]$ & 11.5 & 11.8 & 11.5 & 12.3 & \\
\hline & Sectional obstruction [\%] & 0.8 & 50.1 & 79.4 & 100.0 & \\
\hline \multirow[t]{3}{*}{3} & Balloon diameter [mm] & 2.8 & 12.7 & 15.6 & 18.2 & \multirow[t]{3}{*}{$18 / 40$} \\
\hline & Portal vein diameter [mm] & 17.9 & 17.6 & 17.6 & 18.2 & \\
\hline & Sectional obstruction [\%] & 2.4 & 52.1 & 78.6 & 100.0 & \\
\hline \multirow[t]{3}{*}{4} & Balloon diameter [mm] & 0.2 & 11.7 & 14.7 & 16.8 & \multirow[t]{3}{*}{$18 / 40$} \\
\hline & Portal vein diameter [mm] & 16.0 & 16.2 & 16.1 & 16.8 & \\
\hline & Sectional obstruction [\%] & 0 & 52.2 & 83.3 & 100.0 & \\
\hline
\end{tabular}

PVM with $100 \%$ portal vein obstruction was $82.2 \%$ and $52.6 \%$, respectively (i.e. similar to the values found compared with the initial $0 \%$ occlusion state). Hence, the flow variations found when using the first "initial" $0 \%$ occlusion or the second "control" $0 \%$ occlusion were similar.

PF and PVM were also quantified in the vena cava. These values did not seem to be influenced by the portal occlusion, except for $100 \%$ occlusion measurements for subjects \#2 and \#3. While the flow parameters were stable in the vena cava for subjects \#3 and \#4, their values slowly and regularly decreased for subjects \#1 and \#2.

\subsection{Influence on the liver elasticity}

A good statistical repeatability between the repeated MRE stiffness measurements was observed for the initial $0 \%$, the $80 \%$, the $50 \%$, the second $0 \%$ and the $100 \%$ inflation states, with wCV values (with 95\% lower limit and upper limit confidence intervals) of $4.87 \%$ (2.43\%; $9.78 \%$ ), $4.44 \%$ (2.22\%; 8.89\%), 3.49\% (1.74\%; 7.16\%), 2.67\% (1.32\%; 5.56\%) and 3.72\% (1.86\%; 7.47\%), respectively. In general, the repeatability of the MRE measurement was good, with a wCV general value of 3.90\% (2.85\%; 5.85\%). With wCV values inferior to $7 \%$, these observations are in accordance with the RSNA-QIBA consensus ${ }^{25}$ and tend to reinforce the confidence in the stiffness values, despite the small number of subjects.

Baseline liver stiffness was measured at initial $0 \%$ occlusion as $2268 \pm 131 \mathrm{~Pa}$. Individual evolutions of the liver stiffness are shown in Figure 2 (green dots) against the sectional obstruction of the portal vein. The global evolution of the liver's shear modulus is shown as a function of the portal vein obstruction for the whole cohort as box plots in Figure $3 \mathrm{~A}$. Mean liver softening of $0.8 \% \pm 0.1 \%$ and $7.7 \% \pm 0.4 \%$ were observed for nominal occlusions of $50 \%$ and $80 \%$ of the portal vein, respectively, compared with the initial $0 \%$ occlusion. A mean variation of $12.3 \% \pm 0.9 \%$ (compared with the initial $0 \%$ inflation) to $13.8 \% \pm 1.0 \%$ (compared with the second $0 \%$ inflation) was observed for the $100 \%$ obstruction of the portal vein.

Individual liver elasticities were plotted as a function of the portal PVM (Figure 3B) and PF (Figure 3C) for each nominal portal vein occlusion. Significant Spearman's $r_{s}$ rank correlation was observed between the liver mechanical and portal flow parameters, with the possibility of identifying linear relationships, as attested by the correlation coefficients close to 1 . This relationship was observed for each animal individually, but the limited number of subjects combined with interindividual variations did not allow for a conclusion regarding a significant general relationship between liver mechanical property and portal flow.

\section{DISCUSSION}

This study investigated how liver elasticity can be affected by portal vein obstruction and by the corresponding inflow modifications in an animal model. Previous clinical studies showed that a reduction of the intrahepatic venous blood flow due to PVT results in a significant decrease of the liver stiffness. ${ }^{19,20}$ For instance, a decrease in the liver stiffness of $38 \%$ has been associated with an intrahepatic portal flow decrease of $30 \%$ in the context of the Valsalva maneuver. ${ }^{20}$ In the current preclinical study, the decrease in portal flow was induced by several levels of occlusion of the intraluminal section of the portal vein. The flow parameters were quantified in an original way using 4D-flow MRI with no portal vein 


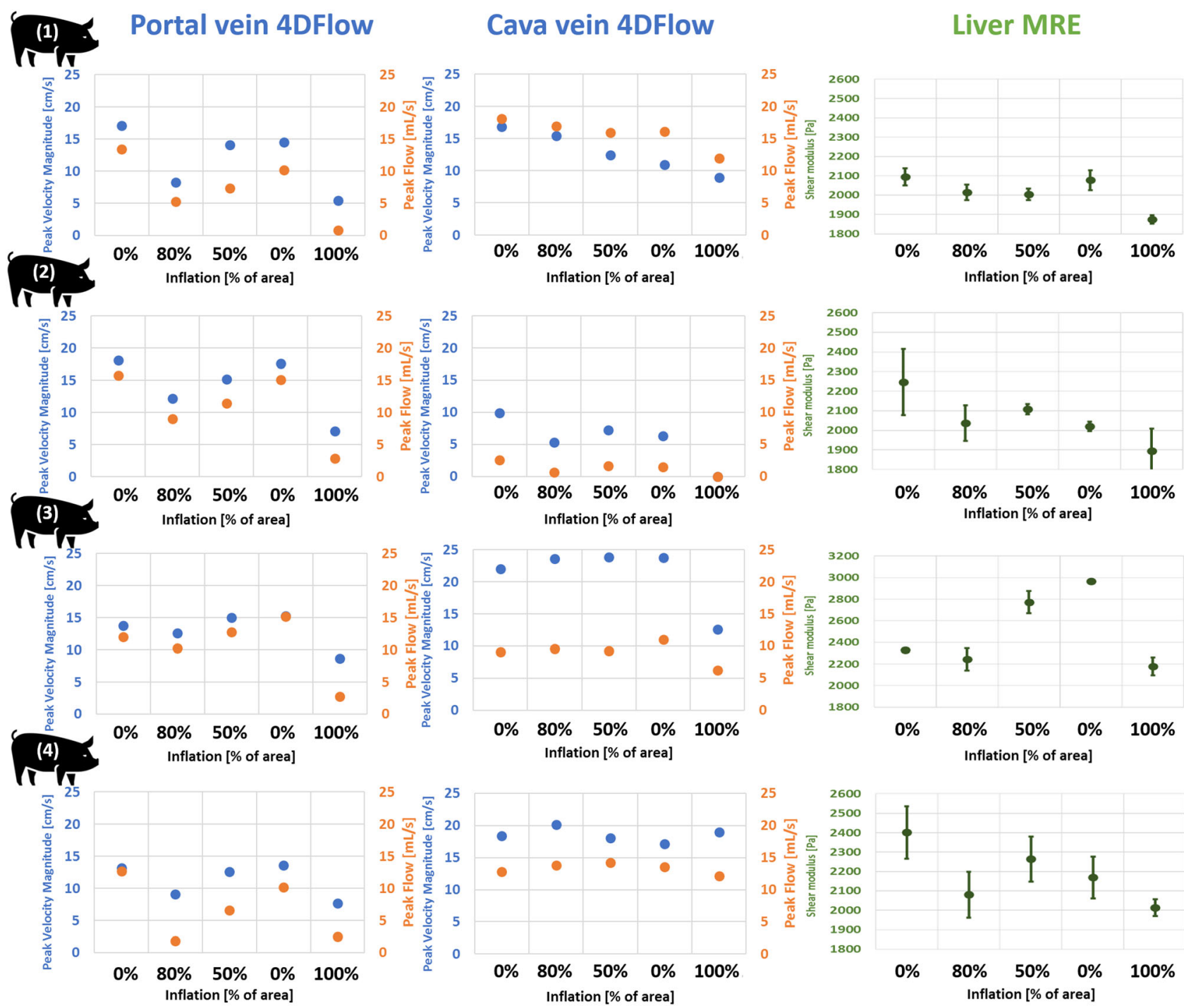

FIGURE 2 Individual evolution of the flow parameters peak velocity magnitude (PVM; blue dots) and peak flow (PF; orange dots) in both the portal (left column) and vena cava (central column) as a function of the portal vein obstruction. Similar evolution was found for the liver stiffness (right column), where the standard deviation bars correspond to variability over the pooled data from the two magnetic resonance elastography (MRE) scans. Note that the shear modulus scale is different in subject \#3 compared with the other subjects

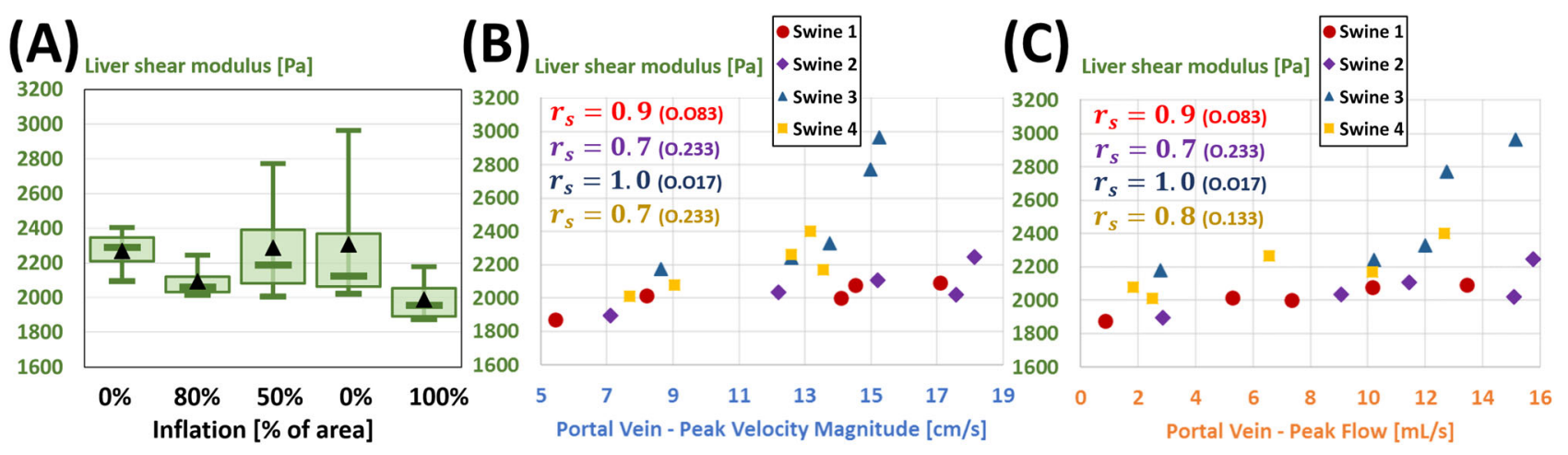

FIGURE 3 Box plots representing the evolution of liver stiffness for the whole cohort with successive degrees of intraluminal portal vein occlusion (A). Liver stiffness is shown as a function of the portal (B) peak velocity magnitude (PVM) and (C) peak flow (PF) for the four subjects. Spearman's $r_{s}$ rank correlation coefficients close to 1 indicate individual monotonic relationships. The level of significance of the correlation is indicated as its $p$-value in the parenthesis 
occlusion and with three levels of portal vein section occlusion (50\%, $80 \%$ and 100\%). The important decreases observed in PF and PVM with portal occlusions were associated with limited decreases in average liver stiffness of $0.8 \%, 7.7 \%$ and $12.3 \%$, respectively, for $50 \%$, $80 \%$ and $100 \%$ portal vein occlusions.

This study has been conducted in swines. The external morphology of the porcine liver is well documented, ${ }^{27}$ however, few studies have detailed the intraparenchymal vascular anatomy compared with humans. Anatomically, the vascular system and the portal system of swine have been found to be similar to the human ones. ${ }^{28}$ The baseline flow values measured in this study in swines were lower (15.53 $\pm 2.45 \mathrm{~cm} . \mathrm{s}^{-1}$ and $13.46 \pm 1.63 \mathrm{~mL} . \mathrm{s}^{-1}$ for PF and PVM, respectively) but in the same range of magnitude as those reported in humans in the literature, with PF of $18.3 \pm 6.7 \mathrm{~mL} . \mathrm{s}^{-129}$ and $19.5 \pm 6.7 \mathrm{~mL} . \mathrm{s}^{-1},{ }^{20}$ and PVM of $22.14 \pm 4.87 \mathrm{~cm} . \mathrm{s}^{-1}$. ${ }^{20}$ Hence, anatomical and portal flow similarities tend to indicate that swine is a valid animal model for vascular effects of PVT on liver elasticity.

The increase of liver stiffness with fibrosis results from a well-known "histological" effect, that is, from modifications in the constitutive hepatic tissue. ${ }^{30}$ For instance, an average increase in liver stiffness of $14 \%$ was reported in a previous study ${ }^{17}$ using MRE between patients without (F0-F1 grades, according to the METAVIR scoring system) and with substantial moderate (F2-F3) fibrosis, while this increase reached 109\% between patients without (FO-F1 grades) and with advanced fibrosis or cirrhosis (F4). In the presence of PVT concomitant with hepatic fibrosis, changes in intrahepatic portal flows may occur due to partial or total occlusion of the portal system. Thus, in accordance with the vascular effects described in this study, these flow changes would cause a decrease in liver stiffness that may attenuate the "histologically" induced increase in stiffness. Given the order of magnitude of the decrease in stiffness caused by PVT-related vascular effects compared with the increase observed with the "histological" effect, ${ }^{16,17}$ portal vascular occlusion is unlikely to have any adverse effect on the diagnosis of elevated fibrosis stages and hepatic cirrhosis by elastography. This confirms the good correlation observed between high fibrosis stage and stiffness measurement. ${ }^{31}$ However, the order of magnitude of the clinical "histological" increase in stiffness reported with moderate stages of fibrosis is comparable with that of the "vascular" decrease in stiffness found in this study in swines. Although the occurrence of concomitant PVT at moderate stages of fibrosis remains low, ${ }^{32}$ there is still a risk of false-negative results in the diagnosis of moderate fibrosis, as recently suggested in a clinical case study. ${ }^{18}$ This tends to suggest a need to couple elastography measurements with PVT evaluation in the detection of early fibrosis based on liver mechanical properties.

The quantifications of PF and PVM in the vena cava do not indicate any influence of the portal occlusion, except for $100 \%$ occlusion measurements for animal subjects \#2 and \#3. For these two subjects, 100\% portal occlusion appeared to have systemic vascular implications, but no conclusions can be drawn because of the limited number of subjects. While the flow parameters were stable in the vena cava for two animal subjects (\#3 and \#4), their values slowly and regularly decreased for the two other subjects (\#1 and \#2), suggesting a potential influence of experimental conditions, such as anesthesia ${ }^{33,34}$ and previous portal vein occlusions. Overall, the systemic vascular variations revealed in the vena cava remain much smaller than those observed in the portal vein with intraluminal occlusions, and their influence on portal flow variations was hence neglected in this work.

It should be noted that subject \#3 gave signs of reawakening within 10 min of the $100 \%$ occlusion, including motion artifacts in MR images. Hence, the isoflurane level was increased, and all acquisitions were repeated for $100 \%$ occlusion once the anesthesia was deep enough. The fact that the anesthesia may have been insufficiently deep could explain why the liver elasticity and flow values measured in this subject for the $50 \%$ and the second $0 \%$ occlusion levels were substantially different from those found in the other subjects. However, no motion artifact was found in the MR images for either $50 \%$ or second $0 \%$ occlusion levels. In the absence of any formal evidence of insufficient anesthesia depth for these two levels, we considered that this subject should not be excluded from this study, but that its values should be considered with care.

Contrary to the increase observed for subject \#3, the decrease observed in liver stiffness for subjects \#1, \#2 and \#4 between the initial and "control" $0 \%$ occlusion levels may be attributed to experimental biases such as the vascular effect of lengthened anesthesia and remnant effects from the two occlusion levels ( $80 \%$ and $50 \%$ ) induced before the second "control" $0 \%$ occlusion level.

This preliminary study was conducted in a limited number of subjects. Please note that, in the absence of prior exclusion criteria, we decided to maintain the data for animal \#3, despite the presence of dextrocardia. A large interindividual variability in the size of the portal vein (diameters from 8.7 to $17.9 \mathrm{~mm}$ for animals of similar weight) was observed. Hence, the nominal balloon diameter was individually adapted to obtain predefined sectional occlusion levels of the portal vein. Despite the limited number of subjects, satisfying repeatability of the MRE measurements was found for each occlusion level according to the RSNA-QIBA consensus. However, interindividual variations and variations between the two $0 \%$ occlusions (i.e. initial and "control") are important and limit the conclusions that can be drawn from this preliminary study. Variations between the two $0 \%$ occlusion levels highlight that the presence of experimental biases, which influence the sensitivity of elasticity measurements, resulted in no change for the $50 \%$ occlusion, while changes in elasticity of $8 \%$ to $12 \%$ were found for the $80 \%$ and $100 \%$ occlusion levels, respectively. Additional work with a larger cohort is required to reinforce the conclusions concerning the effect of partial obstruction of the portal vein on both flow and stiffness measurements. Please note that, in the absence of prior exclusion criteria, we decided to maintain the data for animal \#3 despite the presence of dextrocardia. A large interindividual variability in the size of the portal vein (diameters from 8.7 to $17.9 \mathrm{~mm}$ for animals with similar weight) was observed. Hence, the nominal balloon diameter was individually adapted to obtain predefined sectional occlusion levels of the portal vein. Besides the limited number of subjects, a satisfying repeatability in the evolution of both the flow and mechanical parameters with several levels of portal vein occlusion was found. 
Although evidence of a link between vascular fluid pressure and mechanical properties of the liver has been repeatedly reported, ${ }^{35-40}$ the relationship between vascular flow and hepatic mechanical properties remains poorly described and very rarely mentioned. ${ }^{18}$ In this work, an experimental protocol was proposed to investigate the latter relationship and preliminary results were reported in a limited number of subjects. Further similar investigation is necessary, with the additional measurement of portal pressure or portal resistance, to understand more accurately the mechanisms linking vascular flow, flow velocity and liver mechanical properties. Such an investigation would help to determine whether the acute obstruction represents a typical sinusoidal regulation and the pathophysiologic response of the liver against chronic portal thrombosis. Otherwise, further investigation concerning the kinetics of flow and elasticity changes after the occlusion would be of interest as this point was not investigated in the current study.

In conclusion, this preclinical study illustrated the underlying vascular effect of PVT on liver stiffness measured by elastography. The influence of portal vein obstruction was observed not only on the portal flow PVM and PF with an original use of 4D-flow MRI, but also on liver stiffness using MRE. Results showed a decrease of the liver's shear modulus correlated with the portal flow reduction. This study confirms that vascular effects due to the frequent presence of PVT concomitantly with an acute fibrotic stage or cirrhosis are not likely to alter the diagnosis of the latter by liver elastography. Conversely, moderate fibrosis causes only a slight increase in liver stiffness that may be attenuated in the presence of PVT by the described vascular effects, potentially leading to a false-negative in the diagnosis of moderate fibrosis using elastography. The vascular implications of PVT on liver elasticity reported in this study are of great interest in optimizing the diagnosis of moderate hepatic fibrosis with elastography. Beyond hepatic fibrosis, the influence of blood flow on elastography measurements could be investigated in a similar manner in different organs and pathologies involving both mechanical and vascular modifications, such as polycythemia, certain cancers (liver, pancreas, kidney or adrenal gland), injury or blood clotting disorders.

\section{ACKNOWLEDGEMENTS}

The authors would like to thank Dr. Thomas Benkert (Siemens Healthcare GmbH, Erlangen, Germany) for providing the prototype SPIRAL VIBE sequence, the staff of the imaging platform of the IHU-Strasbourg for their support and availability during the animal experiments and the IRIS platform (ICube laboratory) for technical support. This study received funding from the French state managed by the Agence Nationale de la Recherche (ANR) within the Investissements d'Avenir program for the IHU Strasbourg (Institute of Image Guided Surgery, ANR-10-IAHU-02).

\section{DATA AVAILABILITY STATEMENT}

Data are available on request from the authors.

\section{ORCID}

Simon Chatelin (D) https://orcid.org/0000-0001-8999-3608

Raoul Pop (iD https://orcid.org/0000-0003-4417-1496

Céline Giraudeau iD https://orcid.org/0000-0001-5075-8678

Khalid Ambarki (iD https://orcid.org/0000-0001-7448-8836

François Séverac (1D) https://orcid.org/0000-0002-6611-1921

Elodie Breton (D) https://orcid.org/0000-0003-3614-8069

Jonathan Vappou (D) https://orcid.org/0000-0002-2156-0619

\section{REFERENCES}

1. Ögren M, Bergqvist D, Björck M, Acosta S, Eriksson H, Sternby NH. Portal vein thrombosis: Prevalence, patient characteristics and lifetime risk: A population study based on 23796 consecutive autopsies. World J Gastroenterol. 2006;12(13):2115-2119. https://doi.org/10.3748/wjg.v12.i13.2115

2. Harmanci O, Bayraktar Y. Portal hypertension due to portal venous thrombosis: Etiology, clinical outcomes. World J Gastroenterol. 2007;13(18): 2535-2540.

3. Ponziani FR, Zocco MA, Campanale C, et al. Portal vein thrombosis: Insight into physiopathology, diagnosis, and treatment. World J Gastroenterol. 2010;16(2):143-155.

4. Valla DC, Condat B. Portal vein thrombosis in adults: Pathophysiology, pathogenesis and management. J Hepatol. 2000;32(5):865-871.

5. Okuda K, Ohnishi K, Kimura K, et al. Incidence of portal vein thrombosis in liver cirrhosis: An angiographic study in 708 patients. Gastroenterology. 1985;89(2):279-286.

6. Gaiani S, Bolondi L, Li Bassi S, Zironi G, Siringo S, Barbara L. Prevalence of spontaneous hepatofugal portal flow in liver cirrhosis: Clinical and endoscopic correlation in 228 patients. Gastroenterology. 1991;100(1):160-167.

7. Manzanet G, Sanjuán F, Orbis P, et al. Liver transplantation in patients with portal vein thrombosis. Liver Transpl. 2001;7(2):125-131.

8. Harding DJ, Perera MTPR, Chen F, Olliff S, Tripathi D. Portal vein thrombosis in cirrhosis: Controversies and latest developments. World J Gastroenterol. 2015;21(22):6769-6784.

9. Chawla YK, Bodh V. Portal vein thrombosis. J Clin Exp Hepatol. 2015;5(1):22-40.

10. Zocco MA, Di Stasio E, De Cristofaro R, et al. Thrombotic risk factors in patients with liver cirrhosis: Correlation with MELD scoring system and portal vein thrombosis development. J Hepatol. 2009;51(4):682-689. 
11. Ponziani FR, Zocco MA, Garcovich M, D'Aversa F, Roccarina D, Gasbarrini A. What we should know about portal vein thrombosis in cirrhotic patients: A changing perspective. World J Gastroenterol. 2012;18(36):5014-5020.

12. Foucher J, Chanteloup E, Vergniol J, et al. Diagnosis of cirrhosis by transient elastography (FibroScan): A prospective study. Gut. 2006;55(3):403-408.

13. Sagir A, Erhardt A, Schmitt M, Häussinger D. Transient elastography is unreliable for detection of cirrhosis in patients with acute liver damage. Hepatology. 2008;47(2):592-595.

14. Gómez-Dominguez E, Mendoza J, García-Buey L, et al. Transient elastography to assess hepatic fibrosis in primary biliary cirrhosis. Aliment Pharmacol Ther. 2008;27(5):441-447.

15. Castéra L, Le BB, Roudot-Thoraval F, et al. Early detection in routine clinical practice of cirrhosis and oesophageal varices in chronic hepatitis C: Comparison of transient elastography (FibroScan) with standard laboratory tests and non-invasive scores. J Hepatol. 2009;50(1):59-68.

16. Castera L, Forns X, Alberti A. Non-invasive evaluation of liver fibrosis using transient elastography. J Hepatol. 2008;48(5):835-847.

17. Huwart L, Peeters F, Sinkus R, et al. Liver fibrosis: Non-invasive assessment with MR elastography. NMR Biomed. 2006;19(2):173-179.

18. Huang R, Gao ZH, Tang A, Sebastiani G, Deschenes M. Transient elastography is an unreliable marker of liver fibrosis in patients with portal vein thrombosis. Hepatology. 2018;68(2):783-785.

19. Elkrief L, Rautou P-E, Ronot M, et al. Prospective comparison of spleen and liver stiffness by using shear-wave and transient elastography for detection of portal hypertension in cirrhosis. Radiology. 2015;275(2):589-598.

20. Ipek-Ugay S, Tzschätzsch H, Braun J, Fischer T, Sack I. Physiologic reduction of hepatic venous blood flow by the Valsalva maneuver decreases liver stiffness. J Ultrasound Med. 2017;36(7):1305-1311.

21. O'Donnell M. NMR blood flow imaging using multiecho, phase contrast sequences. Med Phys. 1985;12(1):59-64.

22. R Core Team. R: A language and environment for statistical computing. Vienna, Austria: R Foundation Statistical Computing, 2017.

23. Quan H, Shih WJ. Assessing reproducibility by the within-subject coefficient of variation with random effects models. Biometrics. 1996;52(4): 1195-1203.

24. Shoukri MM, Elkum N, Walter SD. Interval estimation and optimal design for the within-subject coefficient of variation for continuous and binary variables. BMC Med Res Methodol. 2006;6:1-10.

25. RSNA. Quantitative Imaging Biomarkers Alliance. QIBA Profile: Magnetic resonance elastography of the liver. 2018. https://qibawiki.rsna.org/images/ a/a5/MRE-QIBAProfile-2018-05-02-CONSENSUS.pdf. Accessed October 20, 2020.

26. XLSTAT. Data Analysis and Statistical Solution for Microsoft Excel. Paris: Addinsoft; 2017.

27. Couinaud C. Lobes de segments hepatiques: notes sur l'architecture anatomique et chirurgicale du foie. Press Medicale. 1954;62:709-712.

28. Court FG, Wemyss-Holden SA, Morrison CP, et al. Segmental nature of the porcine liver and its potential as a model for experimental partial hepatectomy. Br J Surg. 2003;90(4):440-444.

29. Roldán-Alzate A, Frydrychowicz A, Niespodzany E, et al. In vivo validation of $4 D$ flow MRI for assessing the hemodynamics of portal hypertension. J Magn Reson Imaging. 2013;37(5):1100-1108.

30. Wells RG. Tissue mechanics and fibrosis. Biochim Biophys Acta. 2013;1832(7):884-890.

31. Yin M, Talwalkar JA, Glaser KJ, et al. Assessment of hepatic fibrosis with magnetic resonance elastography. Clin Gastroenterol Hepatol. 2007;5(10): 1207-1213.

32. Papatheodoridis GV, Papakonstantinou E, Andrioti E, et al. Thrombotic risk factors and extent of liver fibrosis in chronic viral hepatitis. Gut. 2003;52 (3):404-409.

33. Gelman S, Fowler KC, Smith LR. Liver circulation and function during isoflurane and halothane anesthesia. Anesthesiology. 1984;61(6):726-730.

34. Kanaya N, Nakayama M, Fujita S, Namiki A. Comparison of the effects of sevoflurane, isoflurane and halothane on indocyanine green clearance. Br J Anaesth. 1995;74(2):164-167.

35. Yin M, Kolipaka A, Woodrum DA, et al. Hepatic and splenic stiffness augmentation assessed with MR elastography in an in vivo porcine portal hypertension model. J Magn Reson Imaging. 2013;38(4):809-815.

36. Ronot M, Lambert S, Elkrief L, et al. Assessment of portal hypertension and high-risk oesophageal varices with liver and spleen three-dimensional multifrequency MR elastography in liver cirrhosis. Eur Radiol. 2014;24(6):1394-1402.

37. Guo J, Büning C, Schott E, et al. In vivo abdominal magnetic resonance elastography for the assessment of portal hypertension before and after transjugular intrahepatic portosystemic shunt implantation. Invest Radiol. 2015;50(5):347-351.

38. Gharib AM, Han MAT, Meissner EG, et al. Magnetic resonance elastography shear wave velocity correlates with liver fibrosis and hepatic venous pressure gradient in adults with advanced liver disease. Biomed Res Int. 2017;2017:1-8.

39. Aquino-Matus J, Uribe M, Chavez-Tapia N. Liver and spleen elastography as predictor of portal hypertension and esophageal varices. Minerva Gastroenterol Dietol. 2020. https://doi.org/10.23736/s1121-421x.20.02779-8

40. Ryu SR, Yoo J-J, Kang SH, et al. The cut-off value of transient elastography to the value of hepatic venous pressure gradient in alcoholic cirrhosis. Clin Mol Hepatol. 2020;27:197-206.

How to cite this article: Chatelin S, Pop R, Giraudeau C, et al. Influence of portal vein occlusion on portal flow and liver elasticity in an animal model. NMR in Biomedicine. 2021;e4498. https://doi.org/10.1002/nbm.4498 